\title{
Studies of the amnesic properties of scopolamine'
}

PETER L. CARLTON AND JOHN R. VOGEL

RUTGERS, THE STATE UNIVERSITY

\begin{abstract}
Prior exposure to a stimulus results in a long-term loss of the effectiveness of that stimulus. Administration of scopolamine before such exposure attenuates this loss of effectiveness in animals subsequently tested without drug. These animals behave much as if they have not had prior exposure at all.
\end{abstract}

\section{Problem}

One of the classical clinical actions of scopolamine is that, in man, it produces amnesia for events that took place when the drug was active. Scopolamine also attenuates the activity of the synaptic transmitter acetylcholine $(\mathrm{ACH})$ in both brain and peripheral nervous system (Goodman \& Gilman, 1955). An obvious supposition based on this fact is that the normal activity of brain-ACH is required for retention; attenuated activity would thus be expected to result in impaired retention.

Our interest in the role of normal ACH-activity in controlling behavior (Carlton, 1963) led to an investigation of the possibility that scopolamine would produce a retention deficit in experimental animals. To do this, we capitalized on the fact that, first, a "novel" stimulus will disrupt the ongoing behavior of an animal and that, second, prior exposure to this stimulus will lead to a loss of its disruptive effect. The major question was whether animals exposed to a stimulus while scopolamine was active would subsequently show this normal effect of exposure when tested in the absence of drug. Or would they behave as if they had not been exposed, i.e., be amnesic?

\section{Method}

\section{Experiment I}

Twelve adult, male, albino rats that had been on a 22-hr. water deprivation regimen for $1 \mathrm{wk}$. were randomly assigned to one of four groups ( $N=3$ / group). A 2 by 2 factorial design was used. One of the variables was exposure to a complex stimulus (S) vs. no stimulus exposure (NS). The other variable was the type of intraperitoneal injection received before each exposure: saline or scopolamine hydrobromide $(0.5 \mathrm{mg} / \mathrm{kg})$. The four groups were thus $\mathrm{S}+$ saline, $\mathrm{NS}+$ saline, $\mathrm{S}+$ scopolamine, and NS + scopolamine.

Fifteen min. following the appropriate injection, each animal was placed in a response chamber from which the levers had been removed. The S groups were intermittently (at variable intervals averaging 1 min.) given 1-min. exposures to a complex stimulus consisting of three elements: a moderate intensity tone (T) at approximately $1000 \mathrm{cps}$, the onset of the houselight in the chamber (HL), and the onset of both cue lights (C) mounted in the front wall of the chamber.

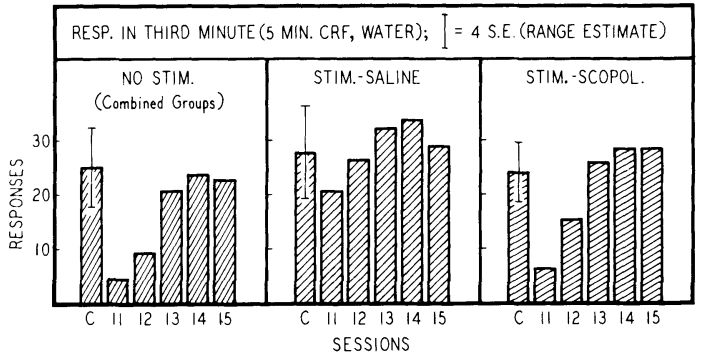

Fig. 1. Numbers of responses during the third min. of 5-min. sessions of CRF. A stimulus was presented during the third min. of sessions 11-15; control values $(C)$ are from sessions 8-10, when no stimulus was presented. Some animals had not had prior exposure (no stim.); others had exposure following injections of either saline or scopolamine (stim.-saline or stim.-scopol.).

The tone and houselight were alternated with the cue lights every 0.25 sec. (i.e., the stimulus sequence was $\mathrm{T}$ and $\mathrm{HL}$ on, $\mathrm{C}$ off $-\mathrm{T}$ and $\mathrm{HL}$ off, $\mathrm{C}$ on-etc.). The NS groups were placed in the chamber but never received stimulus exposure. Seven 20-min. sessions, 3-4 days apart, were given.

Injections and exposure were then discontinued. All animals were dipper-trained (three sessions), the levers were put in place, and all animals were trained on CRF (10 sessions)。

In the third min. of Sessions 11-15, the stimulus was presented to all animals; no injections were given.

\section{Results}

The averaged numbers of responses emitted in the third min. for the various groups are shown in Fig. 1; the data for the two NS groups did not differ and were therefore pooled. The bars marked $\mathrm{C}$ represent the averaged data obtained in Sessions 8-10, when the stimulus was not present. The presentation of the stimulus disrupted the lever-pressing of the NS animals, slightly disrupted the responding of the $\mathrm{S}+$ saline animals, and markedly disrupted the responding of the $\mathrm{S}+$ scopolamine group. All groups recovered to control levels of responding by Session 13 .

Analysis of the numbers of responses emitted in Sessions 11 and 12, the period of depressed responding for the NS animals, indicated that the distribution for the NS animals did not overlap that of the $\mathrm{S}+$ saline animals $(\mathrm{p}=.024$, two-tailed MannWhitney U-test). In contrast, the distribution for the $\mathrm{S}+$ scopolamine group did not differ from the NS distribution $(p=.38)$. Thus, animals given scopolamine prior to exposure behaved as if they had not had' such exposure. 


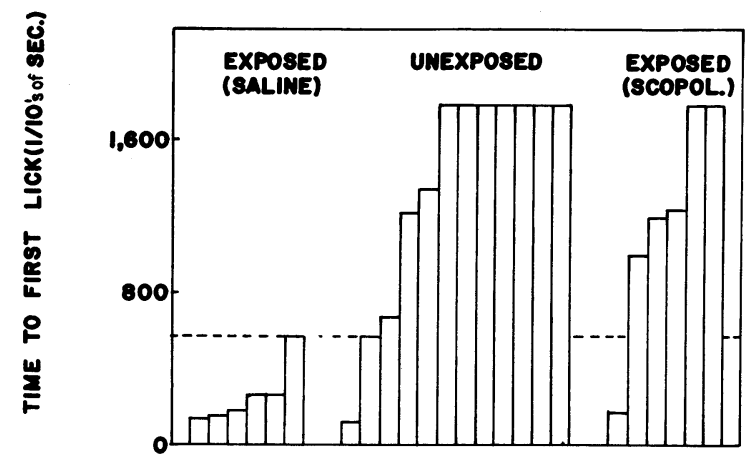

Fig. 2. Time to contact a water-bottle in a 3-min. session. Some animals had not had previous exposure to the drinking-chamber; others had such exposure following injections of either saline or scopolamine. Each vertical bar depicts the result obtained from a single rat; animals failing to contact the bottle were assigned a criterion score of 3 -min.

\section{Method}

\section{Experiment II}

The design and drug-dosage for this experiment were the same as those used for Experiment 1. The two procedural differences were method of exposure and technique for evaluating the effects of that exposure.

Twenty-four rats, maintained on ad lib food and water, were handled for five days. On Day 6 they were placed in one of two chambers for $15 \mathrm{~min}$. The animals were randomly assigned to four groups. Two groups (Exposed-E) were removed from the home cages and placed in the shell (with exhaust fan operating) normally used to house Grason-Stadler response chambers. A small light provided interior illumination. The other two groups (not exposed to the shell-NE) were also removed from their home cages but were placed in a wire cage outside the exposure chamber. All animals were injected (with saline or scopolamine) 20 min. prior to the session on Day 6. There were thus four groups: $\mathrm{E}+$ saline, $\mathrm{E}+$ scopolamine, $\mathrm{NE}+$ saline, and $\mathrm{NE}+$ scopolamine.

A drinking bottle equipped with a drinkometer was then added to the chamber. No treatments were given on Day 7. On Day 8 all animals were water-deprived but were otherwise untreated. On Day 9 all animals were placed in the chamber to which only the $\mathrm{E}$ animals had been exposed on Day 6. No injections were given before this $3-\mathrm{min}$. session. Time required to make initial contact with the water bottle on Day 9 was recorded for each rat; animals that failed to contact the bottle were removed after $3 \mathrm{~min}$.

\section{Results}

The groups that had not had prior exposure to the test situation did not differ. The combined data for these animals have been plotted in the center of Fig. 2; each vertical bar represents the time each animal spent before making initial contact with the water bottle.

The data obtained from the $\mathrm{E}+$ saline animals are at the left of Fig. 1. The horizontal, dashed line denotes the maximum value obtained in this group.

The values for the NE group tend to lie outside the range of the $E+$ saline group $(p=.004)$; i.e., onset of drinking in a "novel" environment was reliably suppressed. In contrast, the $\mathrm{E}+$ scopolamine values are within the distribution of the NE values $(p=.44)$. Animals exposed following scopolamine do not differ from unexposed animals but do differ from those exposed following saline injections $(p=.026)$.

\section{Diseussion}

Both experiments indicate that the loss of stimulus effectiveness consequent to stimulus exposure is substantially reduced by the action of scopolamine at the time of exposure. Animals given exposure following drug-administration behave much as if they have not had such exposure when they are subsequently tested in the absence of the drug.

This result poses at least four questions. First, is the effect due to some kind of "dissociation" (Overton, 1964) or to a stimulus-change between exposure (under drug) and test (no drug)? A second, related question is whether the effects reported here are specific to scopolamine, or will any drug produce this effect?

If the effect proves to be specific to drugs (like scopolamine) known to attenuate the actions of $\mathrm{ACH}$, it would then be reasonable to suppose that the normal activity of $\mathrm{ACH}$ is required for the normal loss of stimulus-effectiveness consequent to exposure. This brings up a third question: does this action of scopolamine reflect the consequence of attenuated $\mathrm{ACH}-$ activity in the brain as opposed to the peripheral nervous system?

Finally, does the effect reported here truly reflect amnesia? The procedures used involved pre-exposure and a resultant loss of stimulus-effectiveness, a loss that is usually assumed to reflect habituation. Does scopolamine produce a general deficit, as the clinical literature suggests, or is the effect peculiar to the habituation process?

\section{References}

Carlton, P.L. Cholinergic mechanisms in the control of behavior by the brain. Psychol. Rer., 1963, 70, 19-39.

Goodman, L. S. \& Gilman, A. The pharmacoloancal basis of therapeutics. New York: Macmillan, 1955

overton, D.A. State-dependent or "dissociated" learning produced with pentobarbital. J. comp. physiol. Psychol., 1964, 57, 3-12.

\section{Note}

1. Research supported by Public Health Service grant MH 08585 